PPPL-2755

UC -427

PPPL-2755

THEORY OF FIELD LINE RESONANCES OF STANDING SHEAR ALFVEN WAVES IN THREE-DIMENSIONAL INHOMOGENEOUS PLASMAS

BY

S. SCHULZE-BERGE, S. COWLEY, AND L. CHEN

May 1991
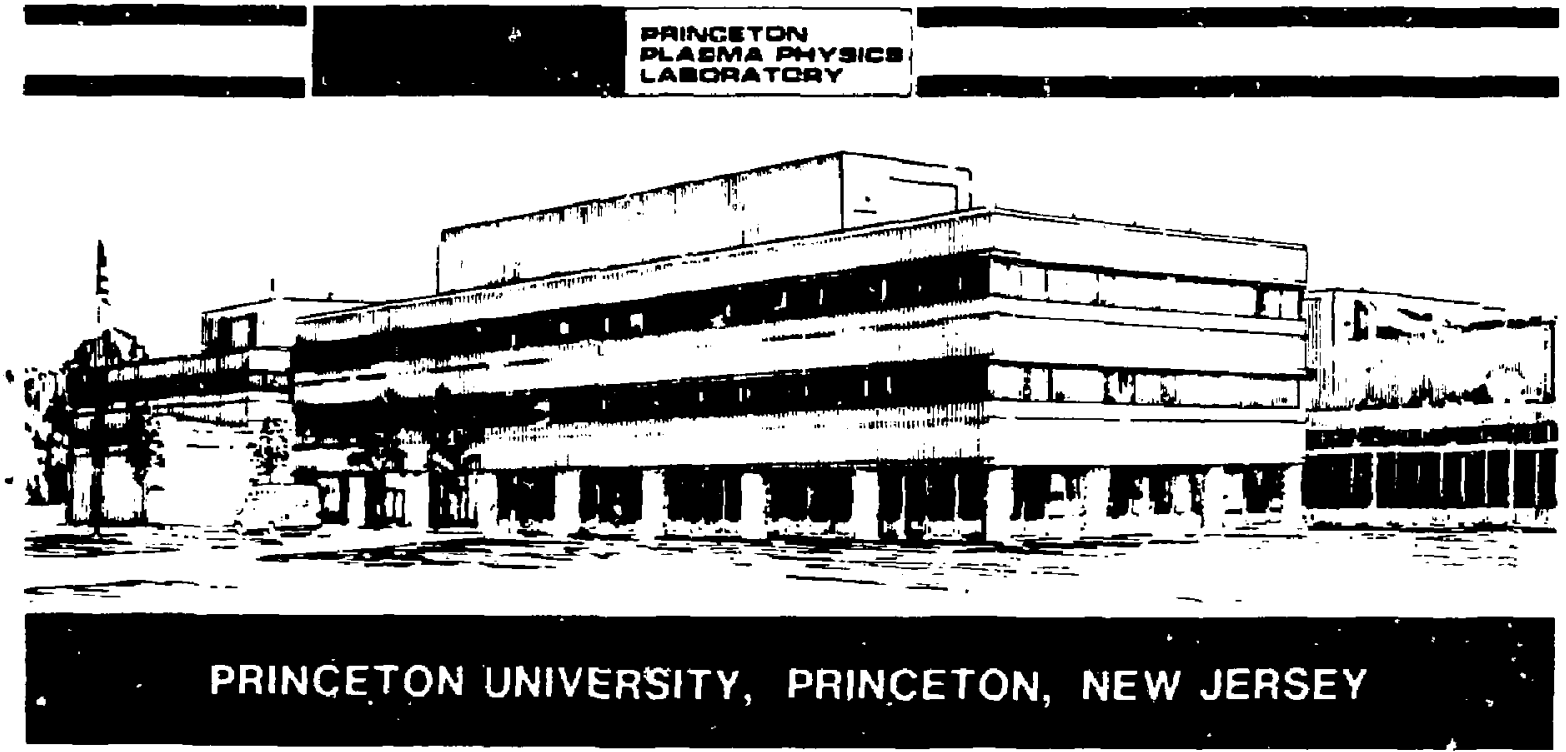


\section{NOTICE}

This report was prepared as an account of work sponsored by an agency of the United States Government. Neither the United States Government nor any agency thereof, nor any of their employees, makes any warranty, express or implied, or assumes any legal liability or responsibility for the accuracy, completeness, or usefulness of any information, apparatus, product, or process disclosed, or represents that its use viould not infringe privately owned rights. Reference herein to any specific commercial produce, process, or service by trade name, trademark, manufacturer, or otherwise, does not necessarily constitute or imply its endorsement, recommendation, or favoring by the United States Government or any agency thereof. The views and opinions of authors expressed herein do not necessarily state or reflect those of the United States Government or any agency thereot.

\section{NOTICE}

This report has been reproduced directly from the best available copy.

Available to DOE and DOE contractors from the:

Office of Scientific and Technical Information

P.O. Box 62

Oak Ridge, TN 37831;

Prices available from (615) 576-8401.

Available to the public from the:

National Technical Information Service

U.S. Department of Commerce

5285 Port Royal Road

Springfield, Virginia 22161

$703-487-4650$ 


\title{
Theory of Field Line Resonances of Standing Shear Alfvén Waves in Three-Dimensional Inhomogeneous Plasmas
}

\author{
Sibylle Schulze-Berge, Steven Cowley, and Liu Chen \\ Princeton Plasma Physics Laboratory and \\ Department of Astrophysical Sciences \\ Princeton University, Princeton, New Jersey 08543
}

\begin{abstract}
We have analyzed field line resonances of Alfven waves in a rectangular box model with a straight uniform magnetic field but three dimensionally varying density. Field line resonances are shown to exist even with this three-dimensional nonuniformity. For a given wave frequency, we can construct the surface on which the resonance occurs and derive the local form of the singular solution. Magnetic perturbations are found to lie predominanty in the resonant surface. In the presence of azimuthal inhomogeneities, the present theory could explain why some satellite measurements show geomagnetic pulsations of comparable magnitude in radial and azimuthal components.
\end{abstract}




\section{Introduction}

The theory of field line resonances (FLR) has been developed in various magnetic field geometries and with different levels of asymmetry. Originally, in the papers of Southwood [1974] as well as Chen and Hasegawa [1974], only one-dimensional inhomogeneity perpendicular to the straight magnetic field $\mathbf{B}$ was assumed. Later inhomogeneities along $\mathbf{B}$ have been accounted for by both Southwood and Kivelson [1986] and Chen and Cowley [1989], the latter using a dipole magnetic field model. As in the original papers, the eigenfrequencies for the transverse (shear) Alfvén waves varied spatially. On the surface where the wave frequency is equal to the shear Alfven frequercy the wave becomes singular. One therefore says that the Alfuen wave is resonant at this surface. The observed spectrum of Alfven waves at a point is expected to be dominated by the resonant component (i.e., the component with the local shear Alfven wave frequency). The perturbed magnetic field will lie predominantly in the azimuthal direction.

All of the models described above neglect nonaxisymmetric inhomogeneities in the magnetosphere such as the day-right asymmetry, the plasma bulge, etc. As it is not clear, how to generalize to these situations without continuous symmetries, we chose to investigate a three-dimensional inhomogeneous plasma in our work. Thus accounting for the previously mentioned azimuthal asymmetries, we examine whether the existence of field line resonances persists in this altered geometry. As in the earlier two-dimensional treatments, the resonant surfaces and the field line eigenfunctions are defined and the form of the singularity is calculated. An additional motivation for introducing a third dimension of asymmetry is given by the observation of field line resonances with $\left|\delta B_{r}\right| \sim\left|\delta B_{\theta}\right|$. A possible explanantion for this kind of satellite measurements is provided.

\section{Theoretical Model}

A cold plasma in a straight uniform magnetic field in $z$ direction will be considered. The density, and therefore the Alfvén speed $v_{A}$, is assumed to vary in $x, y$ and $z$ directions. 
corresponding to the radial, azimuthal, and field-aligned directions in the magnetosphere. The field lines are considered to have fixed end points at $z= \pm \chi_{0}$. No boundary conditions are imposed in $x$ and $y$ directions. We will use ideal magnetohydrodynamic (MHD) equations to describe the plasma, and the perturbed quantities are assumed to vary as $\exp (-i \omega t)$.

Linearizing Ohm's law, force balance, Faraday's law, and the low-frequency Ampere's law and defining $\boldsymbol{\eta} \equiv \xi \times \mathbf{B}$, where $\xi$ is the usual one-fluid displacement vector, we get the following equation

$$
\left\{\frac{\partial^{2}}{\partial z^{2}}+\frac{\omega^{2}}{v_{d}^{2}}\right\} \eta=-\hat{z} \times \nabla \frac{\delta B_{\|}}{B} .
$$

Here

$$
\frac{\delta B_{\|}}{B}=\dot{z} \cdot(\nabla \times \eta)
$$

is the perturbed compressional component of the magnetic field and $v_{A}=v_{A}(x, y, z)=$ $\left[B^{2} / \mu_{0} \rho(x, y, z)\right]^{1 / 2}$ is the Alfvén velocity.

Now replacing $x$ and $y$ with two arbitrary coordinates $p$ and $q$ and with $\nabla p \cdot \nabla q=0$ (we will make an appropriately motivated choice later), we can express $\eta$ as

$$
\eta=\eta_{p} \nabla p+\eta_{q} \nabla q
$$

The Jacobian of this transformation is

$$
J(p, q)=\nabla p \times \nabla q \cdot \hat{z}
$$

Taking the $\nabla p$ and $\nabla q$ components of Eq. (1) and defining $|\nabla q| /|\nabla p|=\Gamma$, the set of equations to be solved is

$$
\begin{gathered}
\frac{\delta B_{\|}}{B}=J(p, q)\left(\frac{\partial \eta_{q}}{\partial p}-\frac{\partial \eta_{p}}{\partial q}\right), \\
\left\{\frac{\partial^{2}}{\partial z^{2}}+\frac{\omega^{2}}{v_{A}^{2}}\right\} \eta_{p}=\Gamma \frac{\partial}{\partial q} \frac{\delta B_{\|}}{B}, \text { and } \\
\left\{\frac{\partial^{2}}{\partial z^{2}}+\frac{\omega^{2}}{v_{A}^{2}}\right\} \eta_{q}=-\frac{1}{\Gamma} \frac{\partial}{\partial p} \frac{\delta B_{\|}}{B} .
\end{gathered}
$$




\section{FLR Eigenfunctions}

Next let us define field line resonance (FLR) eigenfunctions $a_{i}(p, q, z)$ with eigenvalues $\omega_{i}(p, q)$ and standing wave boundary conditions,

$$
a_{i}\left(p, q, z= \pm \chi_{0}\right)=0,
$$

by the equation

$$
\left\{\frac{\partial^{2}}{\partial z^{2}}+\frac{\omega_{i}^{2}}{v_{A}^{2}}\right\} a_{i}(p, g, z)=0,
$$

where the $\omega_{i}$ are functions of $p$ and $q$. Up to now, $p$ and $q$ are not defined yet, but as the operator $\{\cdots\}$ depends on $z$ only, we can solve Eq. (9) in our initial coordinate system and obtain $\omega_{i}(x, y)$ and $a_{i}(x, y, z)$. Once we will have decided on how to choose $p$ and $q$, we then replace $x$ and $y$ by $x(p, q)$ and $y(p, q)$.

Comparing Eq. (9) with Eq. (1) we see that these would be the eigenfunctions if we were able to set $\delta B_{\|}$to zero thes eliminating the compressional Alfvén wave. These $a_{i}$-the shear Alfvén waves with their corresponding eingenfrequencies $\omega_{i}$-provide a convenient base in which to expand $\eta_{p}, \eta_{q}$, and $\delta B_{\|} / B$. Due to the smooth spatial variation of the Alfvén frequency, they form the so-called shear Alfvén continuum. On any given field line, these eigenfunctions are orthogonal, normalizable, and form a complete base, with the orthogonality condition given by

$$
\int_{-x_{0}}^{x_{0}} \frac{a_{i} a_{j}}{v_{A}^{2}(p, q, z)} d z=\delta_{i j}
$$

where $\delta_{i j}$ is the Kronecker delta. Now we can express $\eta_{p}, \eta_{q}$, and $\delta B_{\|} / B$ in terms of $a_{i}$ as:

$$
\begin{aligned}
\eta_{p} & =\sum_{m=0}^{\infty} \lambda_{m}(p, q) a_{m}(p, q, z), \\
\eta_{q} & =\sum_{m=0}^{\infty} \mu_{m}(p, q) a_{m}(p, q, z), \quad \text { and } \\
\frac{\delta B_{\|}}{B} & =\sum_{m=0}^{\infty} \gamma_{m}(p, q) a_{m}(p, q, z) .
\end{aligned}
$$


The coefficients $\lambda_{m}, \mu_{m}$, and $\gamma_{m}$ can be singular, but the FLR eigenfunctions are smoothly varying.

If we express Eq. (6) in terms of Eqs. (11) - (13) and then examine the $a_{i}$-th component, we have

$$
\left(\omega^{2}-\omega_{i}^{2}\right) \lambda_{i}=\Gamma \int_{-x_{0}}^{x_{0}} a_{i} \frac{\partial}{\partial g} \frac{\delta B_{\| 1}}{B} d z .
$$

We see that $\lambda_{i}$ will be singular and there will be a resonance close to a surface where $\omega=\omega_{i}$. Variations across this surface will be much faster than variations along the surface, which now motivates our final choice of $p$ and $q$ : for $\omega \sim \omega_{i}, p$ is chosen so that $\omega_{i}=\omega_{i}(p), q$ will denote the direction of slow variation along the resonant surface. It should be noted that in general $\nabla \omega_{j}$ is not parallel to $\nabla \omega_{i}$ for $j \neq i$. Thereforc, once we have defined $p$ so that $\omega_{i}=\omega_{i}(p)$, the other surfaces will have to be expressed as $\omega_{j}=\omega_{j}(p, q)$ for $j \neq i$. An exception is given by the case when the $z$ dependence of the density san be factored out, i.e., $\rho=\rho_{x y}(x, y) \rho_{x}(z)$.

A frequency $\omega$ matching an eigenfrequency $\omega_{i}$ will excite the corresponding resonant surface which we have now defined to be given by a surface of constant p. Close to this surface we expect energy absoption and mode conversion. The solutions of Eqs. (5) - (7) will exhibit singular behaviour.

In the following, we shall consider one specific $\omega_{i}$ and, for this purpose, we adopt the notation $\omega=\omega_{i}\left(p_{i}\right)$.

\section{Singular Solutions Near the Resonant Surfaces}

In order to examine singular solutions near a resonant surface $\omega=\omega_{1}\left(p_{i}\right)$, we define a smallness parameter $\epsilon$ to be given by $\epsilon \equiv\left|p-p_{i}\right| /\left|p_{i}\right| \ll 1$. Note that now

$$
\left|\frac{\partial}{\partial p}\right| \sim O\left(\frac{1}{\epsilon}\right) \gg 1
$$

and

$$
\left|\frac{\partial}{\partial q}\right| \sim O(1)
$$


Equation (7) and the observation that the compressional Alfven wave can propagate across B suggest the following expansions:

$$
\begin{aligned}
\frac{\delta B_{\|}}{B} & =\quad \frac{\delta B_{\|}^{(0)}}{B}+\epsilon \frac{\delta B_{\|}^{(1)}}{B}+\cdots, \\
\eta_{P} & =\frac{1}{\epsilon} \eta_{p}^{(-1)}+\eta_{p}^{(0)}+\epsilon \eta_{P}^{(1)}+\cdots,
\end{aligned}
$$

and

$$
\eta_{q}=\quad \eta_{q}^{(0)}+\epsilon \eta_{q}^{(1)}+\cdots .
$$

If we look at the lowest order of Eq. (7), the $\epsilon^{-1}$ order,

$$
0=-\frac{1}{\Gamma} \frac{\partial}{\partial p} \frac{\delta B_{\|}^{(0)}}{B},
$$

we see that $\delta B_{\|} / B$, in lowest order, is smooth across the singular surface. This is consistent with the construction of Eq. (17), and none of the $\gamma_{m}$ in Eq. (13) will be singular.

Next let us examine Eq. (6) which can be rewritten as

$$
\left\{\frac{\partial^{2}}{\partial z^{2}}+\frac{\omega^{2}}{v_{A}^{2}}\right\} \eta_{p}=\left\{\frac{\partial^{2}}{\partial z^{2}}+\frac{\omega_{i}^{2}}{v_{A}^{2}}\right\} \eta_{p}+\left\{\frac{\omega^{2}-\omega_{i}^{2}}{v_{A}^{2}}\right\} \eta_{p}=\Gamma \frac{\partial}{\partial q} \frac{\delta B_{\|}}{B} .
$$

Note that close to a resonant surface $p_{i}$, we can make the Taylor expansion

$$
\left(\omega^{2}-\omega_{i}^{2}\right)_{p} \approx\left(p-p_{i}\right) \frac{\partial}{\partial p}\left(\omega^{2}-\omega_{i}^{2}\right)_{p_{i}} \equiv c\left(p-p_{i}\right) .
$$

Now the $\epsilon^{-1}$ order is given by

$$
\left\{\frac{\partial^{2}}{\partial z^{2}}+\frac{\omega_{i}^{2}}{v_{A}^{2}}\right\} \eta_{p}^{(-1)}=0,
$$

from which we see that (we assume a nondegenerate set of eigenfunctions)

$$
\eta_{p}^{(-1)}=\lambda_{i}^{(-1)}(p, q) a_{i}(p, q, z)
$$

The next order is given by

$$
\left\{\frac{\partial^{2}}{\partial z^{2}}+\frac{\omega_{i}^{2}}{v_{A}^{2}}\right\} \eta_{p}^{(0)}+\left\{\frac{\omega^{2}-\omega_{i}^{2}}{v_{A}^{2}}\right\} \eta_{p}^{(-1)}=\Gamma \frac{\partial}{\partial q} \frac{\delta E_{\|}^{(0)}}{B} .
$$


Multiplying Eq. (25) by $a_{m}$ and integrating from $-\chi_{0}$ to $\chi_{0}$ in $d z$, we have to consider two cases seperately:

$$
\begin{array}{ll}
m \neq i & \left(\omega_{i}^{2}-\omega_{m}^{2}\right) \lambda_{m}^{(0)}=\Gamma \int_{-x_{0}}^{x_{0}} a_{m} \frac{\partial}{\partial q} \frac{\delta B_{\|}^{(0)}}{B} d z, \\
m=i & \left(\omega^{2}-\omega_{i}^{2}\right) \lambda_{i}^{(-1)}=\Gamma \int_{-x_{0}}^{x_{0}} a_{i} \frac{\partial}{\partial q} \frac{\delta B_{\|}^{(0)}}{B} d z .
\end{array}
$$

As both $\Gamma$ and $\delta B_{\|} / B$ are nonsingular functions and as $\partial / \partial q$ is operating on the smoothly varying variable, the right-hand side of Eqs. (26) and (27) is nonsingular. Therefore $\lambda_{m}$ will be nonsingular for all $m \neq i$, and $\lambda_{i}$ will be the only singular $\lambda$ as $\left(\omega^{2} \cdots \omega_{i}^{2}\right)$ is small and vanishes on the resonant surface itself. Thus the main contribution to $\eta_{p}=\sum_{m} \lambda_{m} a_{m}$ will come from the $m=i$ term.

Now taking the $\epsilon^{-1}$ order of Eq. (5),

$$
0=J(p, q)\left(\frac{\partial \eta_{q}^{(0)}}{\partial p}-\frac{\partial \eta_{p}^{(-1)}}{\partial q}\right)
$$

and noting that, because of the way we defined the new coordinate system, $\mathrm{J}(p, q)$ does not vanish anywhere, we see that

$$
\left|\frac{\eta_{P}}{\eta_{q}}\right| \sim O\left(\frac{1}{\varepsilon}\right) \gg 1 .
$$

Therefore also $\left|\lambda_{m} / \mu_{m}\right| \sim O(1 / \epsilon)$ and $\mu_{m}$ can be singular only for $m=i$.

If we continue to examine Eqs. (5) - (7) systematically order by order, we find the form of $\lambda_{i}$ and $\mu_{i}$ to be

$$
\begin{aligned}
& \lambda_{i}=\frac{\bar{\lambda}_{i}^{(-1)}}{p-p_{i}}+\bar{\lambda}_{i}^{(0)}+\cdots \text { and } \\
& \mu_{i}=\quad \bar{\mu}_{i}^{(0)}+\bar{\mu}_{i, i n}^{(0)} \ln \left(p-p_{i}\right)+\cdots
\end{aligned}
$$

where, comparing Eqs. (27) and (30) and using Eq. (22),

$$
\bar{\lambda}_{i}^{(-1)}=\left(p-p_{i}\right) \lambda_{i}^{(-1)}=\frac{\Gamma}{c} \int_{-x_{0}}^{x_{0}} a_{i} \frac{\partial}{\partial q} \frac{\delta B_{\|}^{(0)}}{B} d z
$$


and from Eq. (28)

$$
\bar{\mu}_{i, i n}^{(0)}=\frac{\partial \bar{\lambda}_{i}^{(-1)}}{\partial q} .
$$

Here all $\bar{\lambda}_{b}^{a}$ and $\bar{\mu}_{b}^{a}$ are functions of $q$ only. For $m \neq i$, both $\lambda_{m}$ and $\mu_{m}$ will be nonsingular.

We see that both $\eta_{p}$ and $\eta_{q}$ will get singular close to the resonant surface $p_{i}$. As we approach the singularity $\left[\left(p-p_{i}\right) \rightarrow 0\right], \eta_{p}$ will go like $1 /\left(p-p_{i}\right)$, and $\eta_{q}$ will exhibit a logarithmic singularity $\sim \ln \left(p-p_{i}\right)$. In both directions, the eigenfunction $a_{i}$ whose eigenfrequency $\omega_{i}$ matches the incoming frequency $\omega$ will be dominant. Recalling the definition $\boldsymbol{\eta} \equiv \boldsymbol{\xi} \times \mathbf{B}$, we see that $\xi_{q}$, the displacement (or, equivalently, the magnetic perturbation) in the resonant surface, is more singular than $\xi_{p}$, the displacement (magnetic perturbation) perpendicular to the resonant surface. Furthermore, the resonant surface is not in general parallel to the $x-y$ surface. A probe measuring the magnetic perturbations in this geometry will therefore find components in both $x$ and $y$ direction, representing the two projections of $\delta B_{q}$.

Taking the analogy to the Earth's magnetosphere, let us first consider the axisymmetric case [Chen and Cowley, 1989]. Here the most singular magnetic component will be $\delta B_{\theta}$, parallel to the Earth's surface in the azimuthal direction. Now taking into account azimuthal inhomogeneities, a nonaxisymmetric change in density, e.g., due to the plasma bulge or the daynight asymmetry, will cause the resonant surfaces to be tilted with respect to the undisturbed axisymmetric shells. In this case, perturbations measured to be in $r$ and $\theta$ directions are the two components of $\delta B_{\mathrm{q}}$ respectively.

\section{Summary and Discussion}

We have demonstrated analytically that field line resonances of Alfvén waves persist in a three dimensicnally varying density. Although we have simplified the problem by using a rectangular box model with a straight uniform magnetic field, we belicve that our results can be generalized and applied to the Earth's dipole field. The form of the singularity is similar to that of the previous one- and two-dimensional mcdeis. The dispiacement eigenvector is found to still lie predominantly in the resonant surface, but the location of the resonant surface itself 
is now influenced by azimuthal inhomogeneities in the density. Whereas the two-dimensional calculations predict the magnetic perturbation at the resonance to be mainly in the $\theta$ direction, the inclusion of nonaxisymmetric features allows for an additional radial component of the perturbed field. Note that indeed most observations show a dominant $\delta B_{\theta}$ component [Anderson, 1990], but some measurements indicate $\delta B_{\mathrm{r}}$ and $\delta B_{\theta}$ to be of comparable magnitude. Additional insight could be gained by funther analysis of the available data. For example, it could be investigated whether observations of predominantly transverse waves with an appreciable radial component in addition to an azimuthal component occur during periods of either anticipated or observed azimuthal asymmetry in the plasma density. For future work, a more realistic model could consider a dipole magnetic field as well as finite pressure effects, although the physical picture is not expected to be altered by these shanges.

\section{Acknowledgments}

The authors appreciate useful discussions with $\mathrm{K}$. Takahashi and $M$. Engebretson. This work was supported by NSF grant ATM-89-17019 and U.S. Department of Energy Contract DE-AC02-76- 2HO-3073. 


\section{References}

${ }^{1} B$. Anderson, et al. A statistical study of $P_{\mathrm{c}}$ 3-5 pulsations observed by the AMPTE/CCE magneric fields experiment, 1. Occurrence distribution, J. Geophys. Bies. 95 (A7), 10495 (1990).

${ }^{2} \mathrm{~L}$. Chen and $\mathrm{S}$. Cowley, On field line resorences of hydromagnetic A.fuen waves in dipole magnetic field, Geophys. Res. Lett. 16 (8), 895 (1989).

${ }^{3}$ L. Chen and A. Hasegawa, A theory of long period magretic pulsarions, 1. Steady state excitarions of field line resonances, J. Geophiys. Res. 79, 10241974.

${ }^{4}$ D.J. Southwood, Some fertures of fielu li:e resonances in the magnetosphere, Planet. Space Sci. 22, $483(1974)$.

${ }^{5}$ D.J. Southwood and M.S. Kivelson, The effect of parallel inhomogeneity on magnetospheric wave coupling, J. Geophys. Res. 91. (A6), 6871 (1986). 